\title{
Diagnóstico de tuberculose: perspectiva do profissional de enfermagem da atenção primária
}

\author{
Diagnosis of tuberculosis: perspective of primary care nursing professional \\ Diagnóstico de tuberculosis: perspectiva del profesional de la enfermería en la \\ atención primaria
}

Recebido: 05/06/2016 Aprovado: 19/11/2016 Publicado: 01/05/2017

\author{
Mayane Gabrielle Borges Salzani ${ }^{1}$ \\ Sônia Aparecida da Cruz Oliveira ${ }^{2}$ \\ Maria Amélia Zanon Ponce da Rocha ${ }^{3}$ \\ Giselle Juliana de Jesus 4 \\ Claudia Eli Gazetta ${ }^{5}$ \\ Silvia Helena Figueiredo Vendramini ${ }^{6}$ \\ Maria de Lourdes Sperli Geraldes Santos ${ }^{7}$ \\ Thais Oliveira ${ }^{8}$
}

Este estudo tem como objetivo analisar dificuldades no diagnóstico da tuberculose na perspectiva do profissional de enfermagem na atenção primária. Trata-se de um estudo epidemiológico descritivo realizado em 2009 com profissionais de enfermagem. 0 instrumento usado foi o Primary Care Assessment Tool adaptado para tuberculose. Foram analisadas variáveis gerais e de acesso: sexo, profissão, anos na função, remuneração e local de trabalho, conhecimento da doença, convencimento do doente a coletar escarro, preenchimento dos impressos, sobrecarga de trabalho, acesso às informações, atualização, busca de sintomáticos respiratórios, visita domiciliar para entrega do pote de escarro, educação em saúde. Os dados foram armazenados no Software Statistica 8.0 da Statsoft e foi feita uma análise descritiva. Observou-se falta de conhecimento, dificuldade em preencher impressos e convencer o paciente a coletar escarro. A busca ativa é realizada, em detrimento da educação em saúde ou visitas domiciliares para entrega do pote. É necessário investir em educação permanente para profissionais da atenção primária, porta de entrada do sistema de saúde.

Descritores: Tuberculose; Diagnóstico; Serviços de Saúde; Atenção primária à saúde.

This study aims at analysing the difficulties in reaching a tuberculosis diagnostic in the perspective of the primary care nursing professional. It is a descriptive epidemiological study, conducted in 2009 with nursing professionals. The Primary Care Assessment Tool, adapted for tuberculosis, was the instrument used. General and access variables were analyzed: sex, profession, years working in the position, remuneration and workplace, knowledge about the disease, difficulties convincing the patient to collect sputum, or filling in the forms, work overload, access to information, updating, search for respiratory symptoms, home visits to deliver the recipient for sputum collection, health education. Data was stored in the software Statistica 8.0, from Statsoft, and a descriptive analysis was made. The following problems were observed: lack of knowledge, difficulty in filling out forms, and in convincing the patient to collect sputum. Active searches are performed, but not health education actions or home visits to deliver the sputum recipient. It is necessary to invest in continuing education for professionals in primary care, as it is a gateway to the entire health system.

Descriptors: Tuberculosis; Diagnosis; Health services; Primary health care.

Este estudio tiene como objetivo analizar las dificultades en el diagnóstico de la tuberculosis en la perspectiva del profesional de enfermería en la atención primaria. Se trata de un estudio epidemiológico descriptivo realizado en 2009 con profesionales de enfermería. El instrumento utilizado fue el Primary Care Assessment Tool adaptado para tuberculosis. Fueron analizadas variables generales y de acceso: sexo, profesión, años en la función; remuneración y lugar de trabajo; conocimiento de la enfermedad, convencimiento al paciente a recolectar esputo, llenado de formularios, sobrecarga de trabajo, acceso a las informaciones, actualización, búsqueda de síntomas respiratorios, visita a domicilio para la entrega del pote de esputo y educación para la salud. Los datos fueron almacenados en el Software Statistica 8.0 de Statsoft y se realizó un análisis descriptivo. Pudo observarse falta de conocimiento, dificultad en completar los formularios y convencer al paciente para recoger el esputo. La búsqueda activa es realizada en detrimento de la educación en salud o visitas domiciliarias para la entrega del pote. Es necesario invertir en educación permanente para los profesionales de la atención primaria, puerta de entrada al sistema de salud.

Descriptores: Tuberculosis; Diagnóstico; Servicios de salud; Atención primaria de salud.

\footnotetext{
Enfermeira. Especialista em Enfermagem Clinico Cirúrgica na modalidade Residência. SP, Brasil. ORCID: 0000-0003-2942-9364 E-mail: mayane_salzani@hotmail.com.

2 Enfermeira. Especialista em Administração Hospitalar. Especialista em Saúde da Família. Mestre em Enfermagem em Saúde Pública. Professora Adjunta do Departamento de Enfermagem em Saúde Coletiva e Orientação Profissional da Faculdade de Medicina de São José do Rio Preto (DESCOP-FAMERP), São José do Rio Preto, SP, Brasil. ORCID: 0000-0003-2346-8514 E-mail: soniaoliveira@famerp.br.

${ }^{3}$ Enfemeira Doutora em Ciencias da Saúde pelo Programa de Enfermagem em Saúde Pública. Brasil.

${ }^{4}$ Enfermeira, Mestranda pelo Programa de PósGraduação de Enfermagem Fundamental da EERP-USP. Especialista em Doenças Crônicas não transmissíveisSaúde do Adulto e Idoso na modalidade residência pela UNICAMP e Especialista em Enfermagem do Trabalho pela Faculdade de Medicina de São José do Rio Preto- SP, Brasil. Email: giselle.nana@hotmail.com

${ }^{5}$ Enfermeira. Especialista em Geoprocessamento. Mestre em Enfermagem em Saúde Pública. Doutora em Enfermagem. Professora Adjunta do DESCOP-FAMERP. São José do Rio Preto, SP, Brasil. ORCID: 0000-0002-5222-8360 E-mail: claudiaeli@famerp.br.

${ }^{6}$ Enfermeira. Mestre e Doutora em Enfermagem em Saúde Pública. Professora Adjunta do DESCOP-FAMERP, São José do Rio Preto, SP, Brasil. ORCID: 0000-00033061-0355 E-mail: silvia@famerp.br.

7 Enfermeira. Mestre em Ciências da Saúde. Doutora em Enfermagem. Professora Adjunta do DESCOP-FAMERP. São José do Rio Preto, SP, Brasil. ORCID: 00000001-6110-619X E-mail: mlsperli@gmail.com.

${ }^{8}$ Graduanda em Enfermagem - FAMERP, São José do Rio Preto, SP, Brasil. ORCID: 0000-0001-8858-5928 E-mail: thata23.famerp@gmail.com.
} 


\section{INTRODUÇÃO}

A Tuberculose (TB) é uma enfermidade antiga, mantendo-se como um dos maiores males da humanidade no século XXI1-3. Embora a taxa de incidência da doença tenha sido reduzida em $15,4 \%$ (de 42,8 casos por 100 mil habitantes, em 2001, para 36/100.000, em 2011) e a de mortalidade em $23,4 \%$ (de 3,1 óbitos por 100 mil habitantes em 2001, para 2,4/100.000 em 2011), a TB mantém o Brasil entre os 22 países com a mais alta incidência da doença no mundo, sendo ela considerada como a segunda causa de morte por doenças infecciosas e a principal causa de morte em pessoas vivendo com o vírus da imunodeficiência humana (HIV) / síndrome da imunodeficiência adquirida (AIDS) ${ }^{4,5}$.

Segundo estimativas da Organização Mundial de Saúde (OMS), em 2010 houve 8,8 milhões de novos casos de TB, 1,4 milhões de mortes pela doença e foram notificados 5,7 milhões de novos casos e recaídas. Entre 2008 e 2010 foi registrada uma redução no número de casos novos (de 73.673 para 70.601), que deve ser mantido em declínio e reduzido 6 .

0 novo relatório dos objetivos de desenvolvimento para o milênio destacou o Brasil como um dos sete países, entre os 22 responsáveis pelo maior número de casos da doença, a alcançar as metas de redução da incidência de 51,8 para 37 casos por $100 \mathrm{mil}$ habitantes, no período entre 1990 e 2012, reduzindo assim os óbitos relacionados ${ }^{7}$.

A mortalidade por TB vem diminuindo, mas ainda é um número inaceitável para uma doença que pode ser evitada e tem prognóstico de cura ${ }^{8}$. A doença vem sendo diagnosticada tardiamente, em estado avançado e por instituições hospitalares, resultando, muitas vezes, na morte do doente. Pela política nacional de saúde, a responsabilidade do diagnóstico e tratamento da doença é da Atenção Básica, não se restringindo aos conhecimentos médicos.

Desde 2003, a OMS vem demonstrando sua preocupação em relação à qualificação das equipes de saúde para assumirem a responsabilidade pelas ações de controle da
TB e a integração do controle da doença nos serviços de Atenção Primária à Saúde, que representam um dos desafios para o alcance das metas de detecção e cura ${ }^{9}$.

Aos municípios cabe o desenvolvimento de programas de controle da TB, baseando-se nas normas e orientações do Plano Nacional de Controle da Tuberculose (PNCT) em vigor. Para tanto, é enfatizada a necessidade de adequações frente às diversidades locais existentes, principalmente em relação ao Tratamento Supervisionado (TS) ${ }^{10}$.

Quanto aos profissionais de saúde (PS) estes são atores fundamentais na busca ativa dos doentes. 0 vinculo estabelecido entre profissional e usuário favorece a continuidade do cuidado, principalmente quando há conhecimento do papel sócioeconômico-cultural do usuário e de sua família fortalecendo a relação terapêutica ${ }^{11,12}$.

Vários estudos apontam que faltam recursos humanos (RH) capacitados, interesse e motivação pela equipe de saúde, além de haver um número reduzido e uma grande rotatividade de PS, que gera excesso de trabalho nos serviços de saúde (SS); essas são dificuldades encontradas para o controle da doença ${ }^{13,14}$. É necessário conhecer as necessidades do SS para que possam ser implantadas ações de educação permanente, no sentido de instrumentalizar os profissionais de saúde para o diagnóstico, manejo e controle da TB no âmbito da Atenção Primária à Saúde ${ }^{16}$.

Assim, o objetivo deste estudo é analisar dificuldades no diagnóstico da tuberculose na perspectiva do profissional de enfermagem da atenção primária (APS) em São José do Rio Preto/SP.

\section{MÉTODO}

Trata-se de um estudo epidemiológico descritivo, do tipo inquérito, feito com os profissionais de enfermagem de São José do Rio Preto, município que possui uma população de 419.632 habitantes.

O sistema municipal de saúde é composto por 13 UBS, 04 Policlínicas, 11 UBSF, 01 ambulatório de referência para o tratamento da TB, e 01 serviço de 
atendimento especializado em DST/AIDS (SAE), além de 03 hospitais conveniados ao SUS e 03 privados.

Até 2007 as ações de controle de TB eram realizadas de forma centralizada pela equipe especialista do Programa Municipal de Controle da Tuberculose (PCT), depois do que teve início o processo gradual de descentralização para as unidades de APS, e atualmente, o diagnóstico e tratamento dos doentes de TB do município são de responsabilidade das equipes generalistas que atuam na APS, com retaguarda da equipe especializada do PCT. Essa equipe oferece apoio e suporte técnico para as unidades, é responsável pela gestão do programa e pela alimentação dos sistemas de informação da doença, além de oferecer atendimento clínico aos casos mais complexos. A maior parte dos doentes de TB coinfectados pelo HIV/AIDS é acompanhada no serviço de atendimento especializado (SAE) em doenças infectocontagiosas.

Este estudo fez parte do projeto multicêntrico intitulado "Retardo no diagnóstico da tuberculose: análise das causas em diferentes regiões do Brasil" Edital $\mathrm{MCT} / \mathrm{CNPq} / \mathrm{CT}$ Saúde/MS/SCTIE/DECIT no 034/2008, que teve como um de seus objetivos analisar o desempenho dos serviços de atenção básica e pronto atendimento para o diagnóstico da tuberculose.

Neste projeto, foram entrevistados 160 profissionais de saúde que faziam parte da rede de atenção básica de saúde do município, sendo 54 profissionais de cada tipo de serviço de saúde (UBS, UBSF e PA). Os 54 profissionais entrevistados foram divididos de acordo com a proporção da categoria profissional existente em cada unidade. Assim, foram entrevistados 25 médicos, 7 enfermeiros e 22 auxiliares/técnicos em cada tipo de serviços. A seleção das unidades de saúde e dos entrevistados ocorreu por sorteio.

No presente estudo, optou-se por selecionar somente os profissionais de enfermagem (enfermeiros, auxiliares e técnicos de enfermagem) que atuavam nas unidades de APS (UBS e UBSF). Desta forma, a população deste estudo foi composta por 58 profissionais de enfermagem, sendo 14 enfermeiros e 44 auxiliares/ técnicos de enfermagem.

Para as entrevistas, utilizou-se um instrumento de coleta de dados estruturado, elaborado com base no Primary Care Assessment Tool (PCAT), validado para a avaliação dos serviços de atenção primária no Brasil ${ }^{16}$ e adaptado para avaliar a atenção aos doentes de $\mathrm{TB}^{17}$.

0 instrumento foi constituído por variáveis divididas em seções e dimensões. Neste estudo foram utilizadas as variáveis da seção informações gerais (sexo, categoria profissional, há quantos anos trabalha na função, julgamento da remuneração e local de trabalho) e da dimensão acesso ao diagnóstico (dificuldade em relação à falta de conhecimento da doença, dificuldade para convencer o doente a coletar o escarro, dificuldade para preencher os impressos, sobrecarga de trabalho, frequência com que o profissional tem acesso às informações sobre a TB, forma como o profissional se atualiza, realização de busca de sintomáticos respiratórios, visita domiciliar para entrega do pote de escarro, educação em saúde desenvolvida em instituições parceiras, trabalhos educativos sobre a TB no serviço de saúde, frequência de treinamento sobre a TB, e segurança para diagnosticar o usuário suspeito de TB).

Os entrevistados responderam cada pergunta do questionário com respostas dicotômicas (Sim ou Não), abertas ou segundo uma escala de possibilidades preestabelecida - escala de Likert, a cujas respostas se atribuiu um valor entre 1 e 5 , que permitiu registrar o grau de relação de preferência (ou concordância) das afirmações.

Os dados foram armazenados em um banco de dados do Software Statistica 8.0, da Statsoft. Em seguida foram utilizadas técnicas de analise descritiva dos dados (frequência simples e absoluta) e construídos gráficos e tabelas para apresentar os resultados observados.

0 projeto de pesquisa foi submetido ao Comitê de Ética em Pesquisa da Faculdade de 
Medicina de São José do Rio Preto, atendendo às orientações contidas na resolução 196/96 CNS e foi aprovado dentro das normas, conforme protocolo no $\mathbf{3} 747 / 2011$.

\section{RESULTADOS}

Dos 58 profissionais de saúde entrevistados nas UBS e UBSF, $24,14 \%$ eram enfermeiros e $75,86 \%$ auxiliares/técnicos de enfermagem. $\mathrm{Na}$ tabela 1 estão apresentadas características gerais dos profissionais de enfermagem segundo o tipo de serviço em que atuam. Observa-se o predomínio do sexo feminino $(96,55 \%)$ tanto para as UBS quanto para as UBSF. Apenas 3,45\% dos profissionais são do sexo masculino.

Quanto à quantidade de tempo, 58,62\% dos profissionais da UBSF trabalham em sua função de 0 a 5 anos, enquanto nas UBS, um maior tempo de trabalho predomina: a faixa de 5 a 10 anos.

Quanto à remuneração, 62,07\% dos profissionais da UBSF afirmaram ser "boa", enquanto na UBS, 58,62\% afirmaram ser "regular". 62,07\% funcionários da UBSF e $51,72 \%$ da UBS julgaram ser "bom" trabalhar no local.

Tabela 1. Distribuição dos profissionais de enfermagem de acordo com sexo, tempo na função, julgamento da remuneração e do local de trabalho, segundo o tipo de serviço de saúde em que trabalham, São José do Rio Preto, 2009.

\begin{tabular}{llcc}
\hline \multicolumn{1}{l}{ Especificações gerais } & $\begin{array}{c}\text { UBSF } \\
\text { N (\%) }\end{array}$ & $\begin{array}{c}\text { UBS } \\
\text { N (\%) }\end{array}$ \\
\hline Sexo & Feminino & $28(96,55)$ & $28(96,55)$ \\
Tempo há que & Masculino & $1(3,45)$ & $1(3,45)$ \\
trabalha na função & 0 a 5 anos & $17(58,62)$ & $9(31,03)$ \\
& 5 a 10 anos & $8(27,59)$ & $12(41,38)$ \\
& 10 a 15 anos & $3(10,34)$ & $4(13,79)$ \\
Remuneração & 15 a 20 anos & $1(3,45)$ & $4(13,79)$ \\
& Boa & $18(62,07)$ & $8(27,59)$ \\
& Ruim & $1(3,45)$ & $2(6,90)$ \\
Como é trabalhar no & $8(27,59)$ & $17(58,62)$ \\
local & Regular & $2(6,90)$ & $1(3,45)$ \\
& Muito Ruim & $0(0)$ & $1(3,45)$ \\
& Muito Boa & $18(62,07)$ & $15(51,72)$ \\
& Regular & $6(20,69)$ & $9(31,03)$ \\
& Muito Bom & $4(13,79)$ & $4(13,79)$ \\
& Ruim & $1(3,45)$ & $0(0)$ \\
& Muito Ruim & $0(0)$ & $1(3,45)$ \\
\hline
\end{tabular}

Na tabela 2 é apresentada a distribuição por categoria profissional de respostas pertinentes às dificuldades relacionadas à $\mathrm{TB}$ no serviço de saúde.

Observa-se que a maioria dos enfermeiros $(85,71 \%)$ relatam "às vezes" / "quase sempre" faltar conhecimento sobre TB pelos profissionais, enquanto $61,36 \%$ dos auxiliares/ técnicos de enfermagem relatam o mesmo.

Quanto à dificuldade de convencer o paciente a coletar escarro, os enfermeiros $(57,14 \%)$ afirmam que "sempre" / "quase sempre" passam por essa dificuldade, assim como $65,91 \%$ dos auxiliares/técnicos. $57,14 \%$ dos enfermeiros e $84,09 \%$ dos auxiliares/técnicos "sempre" tem dificuldades no preenchimento dos impressos (pedidos de exame).

Há divergência entre enfermeiros e auxiliares/técnicos de enfermagem quanto à sobrecarga de trabalho, já que $57,15 \%$ dos enfermeiros disseram que "nunca" / "quase nunca" têm sobrecarga, embora $72,73 \%$ dos técnicos/auxiliares afirmem que "sempre" / "às vezes" tem sobrecarga de trabalho nos SS. 
Tabela 2. Distribuição de opiniões sobre as dificuldades relacionadas à TB no serviço de saúde em que trabalha, segundo categoria profissional em São José do Rio Preto, 2009.

\begin{tabular}{|c|c|c|c|}
\hline \multicolumn{2}{|l|}{ Dificuldades } & $\begin{array}{l}\text { Enfermeiro } \\
\text { N (\%) }\end{array}$ & $\begin{array}{l}\text { Auxiliar /Técnico } \\
\text { N (\%) }\end{array}$ \\
\hline \multirow{5}{*}{$\begin{array}{l}\text { Falta de } \\
\text { conhecimento sobre } \\
\text { a doença pelos } \\
\text { profissionais de } \\
\text { saúde }\end{array}$} & Sempre & $1(7,14)$ & $13(29,55)$ \\
\hline & Quase sempre & $4(28,57)$ & $15(34,09)$ \\
\hline & Às vezes & $8(57,14)$ & $12(27,27)$ \\
\hline & Quase nunca & $1(7,14)$ & $2(4,55)$ \\
\hline & Nunca & $0(0)$ & $2(4,55)$ \\
\hline \multirow{5}{*}{$\begin{array}{l}\text { Convencer o } \\
\text { paciente a colher } \\
\text { escarro }\end{array}$} & Sempre & $5(35,71)$ & $22(50,00)$ \\
\hline & Quase sempre & $3(21,43)$ & $7(15,91)$ \\
\hline & Às vezes & $5(35,71)$ & $11(25,00)$ \\
\hline & Quase nunca & $1(7,14)$ & $0(0)$ \\
\hline & Nunca & $0(0)$ & $4(9,09)$ \\
\hline \multirow{5}{*}{$\begin{array}{l}\text { Dificuldade para } \\
\text { preencher os } \\
\text { impressos (pedidos } \\
\text { de exame) }\end{array}$} & Sempre & $8(57,14)$ & $37(84,09)$ \\
\hline & Quase sempre & $1(7,14)$ & $4(9,09)$ \\
\hline & Às vezes & $2(14,29)$ & $3(6,82)$ \\
\hline & Quase nunca & $2(14,29)$ & $0(0)$ \\
\hline & Nunca & $1(7,14)$ & $0(0)$ \\
\hline \multirow{5}{*}{$\begin{array}{l}\text { Sobrecarga de } \\
\text { trabalho }\end{array}$} & Sempre & $3(21,43)$ & $15(34,09)$ \\
\hline & Quase sempre & $1(7,14)$ & $1(2,27)$ \\
\hline & Às vezes & $2(14,29)$ & $17(38,64)$ \\
\hline & Quase nunca & $2(14,29)$ & $4(9,09)$ \\
\hline & Nunca & $6(42,86)$ & $7(15,91)$ \\
\hline
\end{tabular}

De acordo com a tabela 3, observa-se que 78,57\% dos enfermeiros "sempre" / "quase sempre" tem acesso às informações sobre TB e 71,43\% utilizam como fonte de informações internet/livro. Quando foi perguntando sobre como o profissional se atualiza, 78,57\% dos enfermeiros disseram que realizam leitura em geral.

Os auxiliares/técnicos $\quad(45,45 \%)$ afirmam "às vezes" terem acesso às informações sobre a doença, e 52,27\% deles usam como fonte de informações internet/livros, e afirmam se atualizar por meio de leituras em geral.

Observa-se na tabela 4 , que $75,86 \%$ dos profissionais de enfermagem da UBSF e
$51,73 \%$ daqueles da UBS "às vezes" / "sempre" conduzem procuras de casos suspeitos de TB. Nas visitas domiciliares (VD), $62,07 \%$ dos profissionais da UBSF "nunca" / "quase nunca" e 75,86\% da UBS "nunca" realizam a entrega do pote para coleta de escarro. Quanto ao quesito educação em saúde desenvolvida em igrejas, associações de bairro e outras para a entrega de pote de escarro, $62,07 \%$ dos profissionais da UBSF e $68,97 \%$ da UBS afirmam "nunca" realizarem essa atividade.

Sobre orientações relacionadas à TB na comunidade, $75,86 \%$ da equipe de enfermagem da UBSF e 58,61\% da UBS afirmam que "às vezes" / "sempre" / "quase 
sempre" as oferecem. Em relação a trabalhos educativos sobre TB, 65,52\% dos funcionários da UBSF "às vezes" e "sempre" os conduzem, enquanto nas UBS, $51,72 \%$ "ás vezes" o faz.

De acordo com a tabela 5, todos os enfermeiros que trabalham na UBSF ou na UBS se sentem seguros para identificar um usuário com suspeita de $\mathrm{TB}$, diferente dos auxiliares/técnicos, dentre os quais somente $72,73 \%$ afirmaram sentir segurança. Quanto a treinamentos sobre TB, $35,71 \%$ dos enfermeiros e $31,82 \%$ dos auxiliares/técnicos de enfermagem passaram por algum que tenha sido realizado no último ano.

Tabela 3. Distribuição de opiniões sobre o acesso às informações sobre TB e à atualização, segundo a categoria profissional. São José do Rio Preto, 2009.

\begin{tabular}{|c|c|c|c|}
\hline \multicolumn{2}{|c|}{ Informações sobre TB } & \multirow{2}{*}{$\begin{array}{l}\text { Enfermeiro } \\
\mathbf{N}(\%) \\
1(7,14)\end{array}$} & \multirow{2}{*}{$\begin{array}{l}\text { Auxiliar /Técnico } \\
\text { N (\%) } \\
20(45,45)\end{array}$} \\
\hline Com que frequência & Às vezes & & \\
\hline $\begin{array}{l}\text { o(a) } \operatorname{Sr}(\mathrm{a}) \text { tem } \\
\text { acesso a }\end{array}$ & Quase sempre & $4(28,57)$ & $7(15,91)$ \\
\hline informações sobre & Sempre & $7(50,00)$ & $12(27,27)$ \\
\hline ТВ & Nunca & $0(0)$ & $2(4,55)$ \\
\hline & Quase nunca & $2(14,29)$ & $3(6,82)$ \\
\hline \multirow[t]{5}{*}{ Especificar } & Educação continuada & $1(7,14)$ & $7(15,91)$ \\
\hline & Revistas & $0(0)$ & $1(2,27)$ \\
\hline & Noticiários & $0(0)$ & $1(2,27)$ \\
\hline & Internet/livros & $10(71,43)$ & $23(52,27)$ \\
\hline & Panfletos/manuais & $3(21,43)$ & $12(27,27)$ \\
\hline \multirow{8}{*}{$\begin{array}{l}\text { Como o(a) } \operatorname{Sr}(a) \text { se } \\
\text { atualiza }\end{array}$} & Educação continuada & $1(7,14)$ & $4(9,09)$ \\
\hline & \multirow{2}{*}{$\begin{array}{l}\text { Não me atualizo por falta de } \\
\text { tempo }\end{array}$} & $0(0)$ & $1(2,27)$ \\
\hline & & $2(14,29)$ & $6(13,64)$ \\
\hline & Cursos & $0(0)$ & $1(2,27)$ \\
\hline & TV & $0(0)$ & $8(18,18)$ \\
\hline & Com o enfermeiro & $0(0)$ & $1(2,27)$ \\
\hline & Faculdade & $11(78,57)$ & $23(52,27)$ \\
\hline & Leitura em geral & & \\
\hline
\end{tabular}


Tabela 4. Distribuição das ações de TB realizadas pelo serviço de saúde em que trabalham, segundo profissionais de enfermagem nas UBSF e UBS. São José do Rio Preto, 2009.

\begin{tabular}{|c|c|c|c|}
\hline \multicolumn{2}{|c|}{ Ações realizadas } & \multirow{2}{*}{$\begin{array}{l}\text { UBSF } \\
\mathbf{N}(\%) \\
7(24,14)\end{array}$} & \multirow{2}{*}{$\begin{array}{l}\text { UBS } \\
\text { N }(\%) \\
2(6,90)\end{array}$} \\
\hline Visitas domiciliares na sua & Às vezes & & \\
\hline área de abrangência para a & Sempre & $3(10,34)$ & $2(6,90)$ \\
\hline entrega de pote para coleta & Quase sempre & $0(0)$ & $0(0)$ \\
\hline \multirow[t]{3}{*}{ de escarro } & Não sabe & $1(3,45)$ & $1(3,45)$ \\
\hline & Nunca & $12(41,38)$ & $22(75,86)$ \\
\hline & Quase nunca & $6(20,69)$ & $2(6,90)$ \\
\hline Educação em saúde & Às vezes & $4(13,79)$ & $5(17,24)$ \\
\hline desenvolvida em igrejas, & Sempre & $2(6,90)$ & $2(6,90)$ \\
\hline associações de bairro e & Quase sempre & $0(0)$ & $0(0)$ \\
\hline outras para a entrega de & Não sabe & $1(3,45)$ & $2(6,90)$ \\
\hline \multirow[t]{2}{*}{ pote para escarro } & Nunca & $18(62,07)$ & $20(68,97)$ \\
\hline & Quase nunca & $4(13,79)$ & $0(0)$ \\
\hline Orientações relacionadas à & Às vezes & $11(37,93)$ & $10(34,48)$ \\
\hline \multirow[t]{5}{*}{ TB na comunidade } & Sempre & $8(27,59)$ & $3(10,34)$ \\
\hline & Quase sempre & $3(10,34)$ & $4(13,79)$ \\
\hline & Não sabe & $2(6,90)$ & $3(10,34)$ \\
\hline & Nunca & $3(10,34)$ & $7(24,14)$ \\
\hline & Quase nunca & $2(6,90)$ & $2(6,90)$ \\
\hline \multirow{6}{*}{$\begin{array}{l}\text { Trabalhos educativos } \\
\text { sobre TB no serviço de } \\
\text { saúde em que trabalha } \\
\text { durante o ano }\end{array}$} & Às vezes & $12(41,38)$ & $15(51,72)$ \\
\hline & Sempre & $7(24,14)$ & $2(6,90)$ \\
\hline & Quase sempre & $2(6,90)$ & $6(20,69)$ \\
\hline & Não sabe & $1(3,45)$ & $0(0)$ \\
\hline & Nunca & $4(13,79)$ & $2(6,90)$ \\
\hline & Quase nunca & $3(10,34)$ & $4(13,79)$ \\
\hline
\end{tabular}

Tabela 5. Distribuição das respostas dos profissionais de enfermagem a respeito da suspeita de TB, e se passaram por treinamento para tal nos últimos anos. São José do Rio Preto, 2009.

\begin{tabular}{|c|c|c|c|}
\hline \multicolumn{2}{|l|}{ Informações sobre TB } & \multirow{2}{*}{$\begin{array}{l}\text { Enfermeiros } \\
\mathbf{N}(\%) \\
(100)\end{array}$} & \multirow{2}{*}{$\begin{array}{l}\text { Técnicos/auxiliares } \\
\mathbf{N}(\%) \\
32(72,73)\end{array}$} \\
\hline $\begin{array}{l}\text { "O Sr (a) se sente } \\
\text { seguro (a) para }\end{array}$ & Sim & & \\
\hline $\begin{array}{l}\text { identificar usuários } \\
\text { com suspeita de TB" }\end{array}$ & Não & $0(0)$ & $12(27,27)$ \\
\hline Treinamento & 1 a 2 anos & $2(14,29)$ & $8(18,18)$ \\
\hline realizado sobre $\mathrm{TB}$ & 3 a 4 anos & $2(14,29)$ & $9(20,45)$ \\
\hline \multirow[t]{3}{*}{ nos últimos anos } & 5 anos ou mais & $1(7,14)$ & $3(6,82)$ \\
\hline & No último ano & $5(35,71)$ & $14(31,82)$ \\
\hline & Não fez & $4(28,57)$ & $10(22,73)$ \\
\hline
\end{tabular}

\section{DISCUSSÃO}

Observou-se que mais da metade dos profissionais de enfermagem são do sexo feminino, trabalham na mesma função há mais de cinco anos, e estão satisfeitos com seu local de trabalho e com sua remuneração.

Ao se analisar as características dos profissionais estudados, encontrou-se o predomínio do sexo feminino confirmando a tendência nacional de feminilização da força de trabalho na área da saúde ${ }^{18}$.

$\mathrm{Na}$ atenção primária não há restrição para o atendimento, ou seja, a população em geral procura o serviço, e pessoas com todo tipo de problema de saúde são atendidas, uma vez que o serviço tem a função de organizar e racionalizar o uso dos demais recursos $^{19}$. Isso pode gerar desconforto para os profissionais, causando sobrecarga de 
funções, uma vez que precisam realizar diversas atividades sem o preparo e o perfil profissional adequados ${ }^{9}$.

Os enfermeiros percebem que a atualização é muito importante, e as exigências são grandes nos dias de hoje devido à renovação e à invenção de tecnologias, que geram novas visões de trabalho da equipe de saúde. 0 profissional de enfermagem tem que levantar suas próprias necessidades e expectativas de trabalho, e assim buscar um suporte teórico para que perceba suas potencialidades e consequentemente suas dificuldades, e assim, possa se adequar a elas no dia a dia, no suporte ao doente ${ }^{20}$.

O tempo de trabalho dos profissionais de enfermagem na atenção básica é variável, sendo que, aqueles que exercem suas funções na UBSF têm menor tempo no cargo que aqueles que estão na UBS. Observa-se essa distribuição de tempo de trabalho devido à descentralização dos serviços de saúde que ocorreu a partir de 1998 em São José do Rio Preto, quando o município assumiu a Gestão Plena do Sistema de Saúde, municipalizando funcionários antigos, que possuíam vínculo com outros entes federativos, e também iniciando o processo de ampliação das contratações ${ }^{21}$.

0 pagamento normalmente é indicado como sendo o elemento de maior insatisfação no trabalho do enfermeiro. 0 que influencia muito no tempo de trabalho é a remuneração desses profissionais, sendo que mais da metade estão satisfeitos com a sua remuneração e com o local em que trabalham ${ }^{22,23}$. Uma consequência disso é um aumento na motivação e no desempenho do trabalho, aliados à vontade de se aprimorar e aprender cada vez mais.

O Ministério da Saúde (MS) recomenda que todas as categorias de profissionais da atenção primária à saúde sejam contempladas com a educação permanente em saúde para um melhor atendimento ao doente de TB. Outros estudos mostram que existe uma preocupação dos profissionais com relação à capacitação para o cuidado em TB, evidenciando ser esta uma ação importante, que deve garantir a compreensão da doença e seus condicionantes, bem como de instrumentos e tecnologias utilizadas para sua prevenção e controle ${ }^{24}$.

A estratégia que o MS recomenda é para que $o$ atendimento ao usuário seja qualificado, pois todos os profissionais que trabalham na atenção primária, desde médicos, agentes comunitários de saúde e equipe de enfermagem têm grande contato com o usuário. Assim, os profissionais podem conseguir passar mais segurança ao doente, a sua família, e ao mesmo tempo identificar casos suspeitos de TB, para realizar a busca ativa de sintomáticos respiratórios (BASR), TS e o preenchimento de impressos necessários.

Apesar de ser um procedimento simples, a BASR no controle da TB caracteriza-se por uma prática que requer habilidade, pois o contato inicia-se na unidade de saúde e a formação do vínculo vai sendo cada vez maior, pois o profissional tem que estar disposto a inteirar o doente quanto à doença propriamente dita, dando informações sobre sinais e sintomas, orientações sobre a coleta de escarro, o fluxo dos exames laboratoriais e até mesmo encaminhá-lo para outro serviço se for necessário. Assim, a necessidade de investir na qualificação dos recursos humanos é urgente e deve ser uma ação contínua, para melhores condições de trabalho e de resolutividade dos serviços 25 .

Quanto às ações desenvolvidas pelos serviços de saúde em que trabalham, tanto os profissionais de enfermagem da UBSF quanto os da UBS encontram-se em divergência, já que a maioria relata que sempre realizam BASR para TB em suas unidades, ainda que os resultados mostrem que nunca ou quase nunca realizam VD e/ou educação em saúde em igrejas e/ou associações de bairro para entrega do pote para coleta de escarro.

Verifica-se que o desempenho dos serviços na assistência aos doentes de TB ainda está pouco orientado para a comunidade e para as reais necessidades de saúde da população, apontando a necessidade de se repensar a prática, de uma atuação que extrapole os "muros" dos serviços de saúde. A realização de visitas 
domiciliares é necessária no contexto social do doente e de sua família, estabelecendo assim um vínculo entre eles. 0 profissional da saúde está diretamente ligado ao doente, principalmente a equipe de enfermagem, que tem um contato mais prolongado. Esse vínculo possibilita conhecer melhor o ambiente no qual os mesmos estão inseridos, suas necessidades e particularidades, possibilitando assim acompanhar o tratamento do doente e seus familiares ${ }^{2}$.

Quanto a ter acesso a informações sobre $\mathrm{TB}$, o que utilizam como fonte de informações e como se atualizam, ambas as categorias foram unânimes em responder que têm acesso a essas informações por meio de internet/livros e que se atualizam por meio de leituras em geral. Essa dificuldade pode estar relacionada à falta de capacitação para os profissionais.

Estudos realizados no Brasil demonstraram que além da falta de conhecimento sobre o manejo da TB pelos PS, também se constatou a falta de planejamento e treinamento em serviço ${ }^{13,26,27 .}$

Em São José do Rio Preto, foi identificado que os PS não eram capacitados adequadamente em relação ao PCT e também havia dificuldades em deslocá-los do ambiente de trabalho para receberem treinamento, em função de outras prioridades locais. A dificuldade no acesso do usuário e consequente demora na efetivação do diagnóstico de TB, não é uma exclusividade do município, mas sim algo presente em diversas regiões do mundo ${ }^{28}$.

As falhas no processo de capacitação dos PS, aliadas à rotatividade dos mesmos, faz com que não sejam efetivos para a manutenção dos conhecimentos e, consequentemente, do diagnóstico da TB.

Assim, se tornam necessárias estratégias para a qualificação e capacitação permanente dos PS que atuam em serviços de atenção básica. Essa necessidade pode estar conectada à demora no acesso ao diagnóstico da doença, e acontece também com outros tipos de patologias, pois com os sistemas de saúde fragmentados há predominância do atendimento para condições agudas das doenças, deixando para trás as que estão em fase crônica ${ }^{29}$.

Segundo estudo realizado em Fortaleza $^{30}$, o treinamento e sensibilização da equipe de ESF foram capazes de promover o aumento da detecção de casos de TB, ou seja, a atualização e manutenção da equipe treinada motiva os profissionais, e com isso, há diagnóstico e tratamento antecipado, o que contribui para o controle da doença.

\section{CONCLUSÃO}

Com vistas aos objetivos propostos, as principais dificuldades apontadas pelos profissionais de enfermagem foram falta de conhecimento sobre TB, problemas para convencer o paciente a coletar o escarro e preencher o pedido de exame, sobrecarga de trabalho, principalmente entre os técnicos, e dificuldades na busca ativa de sintomáticos respiratórios, principalmente fora da UBS e nas visitas domiciliares. Outra dificuldade apontada foi com relação à educação em saúde e educação permanente.

Esses resultados remetem à necessidade de investir mais em estratégias que visem a qualificação e capacitação permanente e sistematizada dos profissionais de enfermagem, bem como de outras categorias que atuam nos diversos serviços que se constituem como porta de entrada da rede de atenção primária à saúde.

\section{REFERÊNCIAS}

1. World Health Organization. WHO report 2011: global tuberculosis control. Geneva: World Health Organization; 2011.

2. Ministério da Saúde (Br). Secretaria de Vigilância em Saúde. O controle da tuberculose no Brasil: avanços, inovações e desafios. Bol Epidemiol. [Internet]. 2014 [citado em 15 nov 2012]; 45(2):1$13 . \quad$ Disponível em: http://portalsaude.saude.gov.br/images/pdf/2014 /maio/29/BE-2014-45--2--tb.pdf.

3. Ministério da Saúde (Br). Secretaria de Vigilância em Saúde. Especial tuberculose. Bol Epidemiol. [Internet]. 2012 [citado em 29 nov 2012]; 43:1-12. Disponível em: http://portalsaude.saude.gov.br/images/pdf/2014 /julho/23/BE-2012-43-Mar--o---Especial-

Tuberculose.pdf.

4. Muniz JN, Rufino Netto A, Villa CS, Yamamura M, Arcencio R, Cardozo-Gonzales RI. Aspectos epidemiológicos da co-infecção tuberculose e vírus 
da imunodeficiência humana em Ribeirão Preto (SP), de 1998 a 2003. J Bras Pneumol. [Internet]. 2006 [citado em 19 nov 2013]; 32(6):529-34. Disponível

em:

http://www.scielo.br/pdf/jbpneu/v32n6/a10v32n

6.pdf. DOI: http://dx.doi.org/10.1590/S180637132006000600010.

5. Villa TCS, Ponce MAZ, Wysock AD, Andrade RLP, Arakawa T, Scatolin BE, et al. Diagnóstico oportuno da tuberculose nos serviços de saúde de diversas regiões do Brasil. Rev Latinoam Enferm. [Internet]. 2013 [citado em 02 nov 2011]; 21(esp):[08 telas]. Disponível em: http://www.scielo.br/pdf/rlae/v21nspe/pt_24.pdf. DOI: http://dx.doi.org/10.1590/S0104-

11692013000700024.

6. World Health Organization. Global tuberculosis report 2012. [Internet]. Geneva: WHO; 2012. [citado em 28 jun 2016]. Disponível em: http://apps.who.int/iris/bitstream/10665/75938/ 1/9789241564502_eng.pdf.

7. Presidência da República (Brasil). Objetivos de desenvolvimento do milênio: relatório nacional de acompanhamento [Internet]. Brasília, (DF): IPEA; 2014 [citado em 12 nov 2012]. 208p. Disponível em:

http://www.ipea.gov.br/portal/images/stories/PD Fs/140523_relatorioodm.pdf.

8. Yamamura M, Zanoti MDU, Arcêncio RA, Protti ST, Figueiredo RM. Mortalidade por tuberculose no interior de São Paulo-Brasil (2006-2008). Ciênc Cuid Saúde. 2015; 14(3):1259-65.

9. Monroe AA, Cardozo Gonzales RI, Palha PF, Sassaki CM, Ruffino Netto A, Vendramini SHF, et al. Envolvimento de equipes da atenção básica à saúde no controle da tuberculose. Rev Esc Enferm USP. [Internet]. 2008 [citado em 20 ago 2012]; 42(2):262-7. Disponível em: http://www.scielo.br/pdf/reeusp/v42n2/a07.pdf. DOI: $\quad$ http://dx.doi.org/10.1590/S008062342008000200008.

10. Protti ST, Silva LMC, Palha PF, Villa TCS, RuffinoNeto A, Nogueira JA, et al. A gerência da unidade básica de saúde no controle da tuberculose: um campo de desafios. Rev Esc Enferm USP. [Internet]. 2010 [citado em 16 dez 2012]; 44(3):66570. Disponível em: http://www.scielo.br/pdf/reeusp/v44n3/16.pdf. DOI: $\quad$ http://dx.doi.org/10.1590/S008062342010000300016.

11. Souza KMJ, Sá LD, Palha PF, Nogueira JA, Villa TCS, Figueiredo DA. Abandono do tratamento de tuberculose e relações de vínculo com a equipe de saúde da família. Rev Esc Enferm USP. [Internet]. 2010 [citado em 28 out 2012]; 44(4):904-11. Disponível em: http://www.scielo.br/pdf/reeusp/v44n4/07.pdf. DOI: $\quad$ http://dx.doi.org/10.1590/S008062342010000400007.
12. Sousa TF, Souza FBA, Carvalho FC, Villa TCS, Ruffino-Netto, A. Estratégia saúde da família e a construção do vínculo no controle da tuberculose pulmonar na Rocinha-RJ. Rev Pesqui Cuid Fundam. [Internet]. 2015 [citado em 14 nov 2012]; 7(2):2336-2348. Disponível em: http://seer.unirio.br/index.php/cuidadofundament al/article/view/3562/pdf_1543. http://dx.doi.org/ 10.9789/2175-5361.2015.v7i2.2336-2348.

13. Ponce MZ, Wysocki AD, Scatolin BE, Andrade RLP, Arakawa T, Ruffino Netto A, et al. Diagnóstico da tuberculose: desempenho do primeiro serviço de saúde procurado em São José do Rio Preto, São Paulo, Brasil. Cad Saúde Pública. [Internet]. 2013 [citado em 22 jan 2013]; 29(5):945-54. Disponível em: http://www.scielo.br/pdf/csp/v29n5/12.pdf. DOI: $\quad$ http://dx.doi.org/10.1590/S0102311X2013000500012.

14. Balderrama P, Vendramini SF, Santos MLSG, Ponce MAZ, Oliveira IC, Villa TCS, et al. Porta de entrada para o diagnóstico da tuberculose: avaliação da estrutura dos serviços. Rev Eletrônica Enferm. [Internet]. 2014[citado em 22 nov 2012]; 16(3):511-9. Disponível em: http://revistas.ufg.br/fen/article/view/21408/175 41. DOI: http://dx.doi.org/10.5216/ree.v16i3.21408. 15. Antunes LB, Tomberg JO, Harter J, Lima LM, Beduhn DAV, Gonzales RIC. Sintomático respiratório de tuberculose na atenção primária: avaliação das ações segundo as recomendações nacionais. Rev RENE. 2016; 17(3):409-15.

16. Macinko J, Almeida C. Validação de uma metodologia de avaliação rápida das características organizacionais e do desempenho dos serviços de atenção básica do sistema único de saúde (SUS) em nível local. Brasília: Organização Pan-Americana da Saúde; 2006.

17. Villa TCS, Ruffino-Netto A. Questionário para avaliação de desempenho de serviços de atenção básica no controle da tuberculose no Brasil. J Bras Pneumol. [Internet]. 2009 [citado em 22 nov 2012]; 35(6):610-2. Disponível em: http://www.scielo.br/pdf/jbpneu/v35n6/v35n6a1 4.pdf. DOI: http://dx.doi.org/10.1590/S180637132009000600014.

18. Ferreira MBG, Felix MMS, Contim D, Souza DJ, Simões ALA. Caracterização do contexto de trabalho e qualidade de vida dos profissionais de estratégia saúde da família. Cogitare Enferm. 2015; 20(3):56572.

19. Lopes LMG, Vieira NF, Lana FCF. Análise dos atributos da atenção primária à saúde na atenção à tuberculose no Brasil: uma revisão integrativa. Rev Enferm Cent-Oeste Min. 2015; 5(2):1684-1703.

20. Sá LD, Gomes ALC, Carmo JB, Souza KMJ, Palha $\mathrm{PF}$, Alves RS, et al. Educação em saúde no controle da tuberculose: perspectiva de profissionais da estratégia Saúde da Família. Rev Eletrônica Enferm. 
[Internet]. 2013 [citado em 22 nov 2013]; 15(1):103-11. Disponível em: https://www.fen.ufg.br/fen_revista/v15/n1/pdf/v 15n1a12.pdf. http://dx.doi.org/10.5216/ree.v15i1.15246.

21. Vendramini SHF. O programa de controle da tuberculose em São José do Rio Preto-SP: do contexto epidemiológico à dimensão social - 2005. [tese]. Ribeirão Preto: Escola de Enfermagem de Ribeirão Preto, Universidade de São Paulo; 2005. 259p.

22. Morais MP, Martins JT, Galdino MJQ, Robazzi MLDCC, Trevisan GS. Satisfação no trabalho de enfermeiros em um hospital universitário. Rev Enferm UFSM. 2016; 6(1):1-9.

23. Sá LD, Oliveria AAV, Gomes ALC, Nogueira JA, Villa TCS, Collet N. Cuidado ao doente de tuberculose na estratégia saúde da família: percepções de enfermeiras. Rev Esc Enferm USP. [Internet]. 2012 [citado em 22 nov 2012]; 46(2):356-63. Disponível em: http://www.scielo.br/pdf/reeusp/v46n2/a13v46n 2.pdf. DOI: http://dx.doi.org/10.1590/S008062342012000200013.

24. Silva-Sobrinho RA, Zilly A, Monroe AA, Pinto ESG, Silva RMM, Villa TCS. Ambivalência das ações de controle da tuberculose na atenção básica à saúde. Rev RENE. 2014; 15(4):605-12. 25. CardozoGonzales RI, Palha PF, Harter J, Alarcon E, Lima LM, Tomberg JO. Avaliação das ações de detecção de casos de tuberculose na atenção primária. Rev Eletrônica Enferm. [Internet]. 2015; 17(4):1-8. Disponível em: http://revistas.ufg.br/fen/article/view/32846/206 97. DOI:

http://dx.doi.org/10.5216/ree.v17i4.32846.

26. Dantas DNA, Enders BC, Queiroz AAR, Coura AS, Silva MPM, Menezes RMP. Fatores associados à primeira escolha de local para o diagnóstico da tuberculose. Rev Gaúch Enferm. 2014; 35(3):75-81. 27. Santos MLSG, Villa TCS, Vendramini SHF, Gonzáles RIC, Palha PF, Santos NSGM, et al. Gerência das ações de controle da tuberculose em municípios prioritários do interior paulista. Texto \& Contexto Enferm. [Internet]. 2010 [citado em 28 nov 2012]; 19(1):64-9. Disponível em: http://www.scielo.br/pdf/tce/v19n1/v19n1a07.pd f. DOI: http://dx.doi.org/10.1590/S010407072010000100007.

28. Nogueira JA, Anjos UU, Sá LD, Silva DM, Trigueiro, DRSG, Villa TCS, et al. A influência dos determinantes individuais no retardo do diagnóstico da tuberculose. Texto \& Contexto Enferm. [Internet]. 2014 [citado em 10 fev 2016]; 23(4):1022-31. Disponível em: http://www.redalyc.org/articulo.oa?id=714335080 25. DOI: http://dx.doi.org/10.1590/010407072014001370013.

29. Mendes EV. As redes de atenção à saúde. Ciênc Saúde Coletiva. [Internet]. 2010 [citado em 25 fev 2013]; 15(5):2297-305. Disponível em: http://www.scielo.br/pdf/csc/v15n5/v15n5a05.pd f. DOI: http://dx.doi.org/10.1590/S141381232010000500005.

30. Façanha MC, Melo MA, Vasconcelos FF, Souza JRP, Pinheiro AS, Porto IA, et al. Treinamento da equipe de saúde e busca ativa na comunidade: estratégias para a detecção de casos de TB. J Bras Pneumol. [Internet]. 2009 [citado em 22 nov 2012]; 35(5):449-54. Disponível em: http://www.scielo.br/pdf/jbpneu/v35n5/v35n5a1 0.pdf. DOI: http://dx.doi.org/10.1590/S180637132009000500010 .

\section{CONTRIBUIÇÕES}

Todas as autoras tiveram iguais contribuições no desenho do estudo, análise e redação final e crítica do artigo.

\footnotetext{
Como citar este artigo (Vancouver)

Salzani MGB, Oliveira SAC, Rocha MAZP, Jesus GJ, Gazetta CE, Vendramini SHF, Santos MLSG, Oliveira T. Diagnóstico de tuberculose: perspectiva do profissional de enfermagem da atenção básica. REFACS [Internet]. 2017 [citado em dia, mês e ano de acesso]; 5(2):180-190. Disponível em: link de acesso. DOI:.

\section{Como citar este artigo (ABNT)}

SALZANI, M. G. B. et al. Diagnóstico de tuberculose: perspectiva do profissional de enfermagem da atenção básica. REFACS, Uberaba, MG, v. 5, n. 2, p. 180-190, 2017. Disponível em: <link de acesso>. Acesso em: inserir dia, mês e ano de acesso. DOI:.

\section{Como citar este artigo (APA)}

Salzani, M. G. B., Oliveira, S. A. C., Rocha, M. A. Z. P., Jesus, G. J., Gazetta, C. E., Vendramini, S. H. F., Santos, M. L. S. G. \& Oliveira, T. (2017). Diagnóstico de de tuberculose: perspectiva do profissional de enfermagem da atenção básica. REFACS, 5(2), 180-190. Recuperado em inserir dia, mês e ano de acesso. Inserir link de acesso. DOI:.
} 